\title{
Lithium abundances in southern associations containing young stars
}

\author{
Licio da Silva ${ }^{1}$, Carlos Alberto Torres ${ }^{2}$, Ramiro de la Reza ${ }^{1}$, Germano \\ Quast $^{2}$, Claudio de Melo ${ }^{3}$, and Michael Sterzik ${ }^{4}$ \\ ${ }^{1}$ Observatório Nacional, Brazil ${ }^{1}$ \\ email: licio@on.br \\ ${ }^{2}$ Laboratório Nacional de Astrofísica, Brazil \\ ${ }^{3}$ European Southern Observatory, Germany \\ ${ }^{4}$ European Southern Observatory, Chile
}

Keywords. stars: pre-main-sequence, stars: evolution, stars: abundances

\section{Introduction}

In a recent paper, da Silva et al. (2009), we report results of $\mathrm{Li}$ abundance analysis for nine young stellar associations, defined in Torres et al. (2008), from a high-resolution optical spectroscopic survey searching for associations containing young stars (SACY), among optical counterparts of ROSAT All-Sky X-ray sources in the Southern Hemisphere. They have applied a convergence method in the (UVW) velocity space and have determined nine nearby young associations in the sample. As they are young and with different ages, those associations form an interesting laboratory to test the Li depletion theory, as a function of the star age.

\section{Analysis}

For this research, most of the spectroscopic observations were performed with the highresolution (RP 50000) FEROS spectrograph Kaufer et al. (1999) at the 1.5m ESO telescope at La Silla (Chile). A smaller set of data $(\sim 30 \%)$ was collected at the coudé spectrograph attached to the $1.60 \mathrm{~m}$ telescope at the Observatório do Pico dos Dias, LNA, Brazil, with a RP 9000 . To obtain the Li abundance we used a LTE code and the atmospheric models of Kurucz and Castelli (www.user.oat.ts.astro.it/castelli). A larger part of the stars effective temperatures Teff were determined from the $\left(\mathrm{V}-\mathrm{I}_{c}\right)$ color index. The other atmosphere model parameters, not determined by us, were fixed a priori: the metalicity as 0.1 and $\log \mathrm{g}$ as 4.5 , for dwarfs, and 4.0, for sub-giants. The used spectral classification is that of Torres et al. (2008). The microturbulence velocity also was fixed as $2 \mathrm{~km} / \mathrm{s}$. We presented in da Silva et al. (2009) our determination of $\mathrm{Li}$ abundance for nine associations, discovered or better determined in the SACY analysis, the associations of $\epsilon$ Chamaleontis $(\epsilon \mathrm{ChA})(6,6)$, TW Hydrae (TWA) $(8,9), \beta$ Pictoris $(\beta$ PA) $(10$, 1), Octans (OctA)(20?,8), Tucana-Horologium (THA)(45, 7), Columba (ColA)(30, 4), Carina $(\mathrm{Car} A)(30,5)$, Argus (ArgA) $(40,3)$, AB Doradus (ABDA)(70,2). The numbers between parenthesis are the association age in My and its number on Fig. 1, respectively. The main data of those associations (i.e., number of members, mean distance and age) were determinated by Torres et al. (2008). The results for those associations presented in da Silva et al. (2009) are final but for ABDA and ColA. These two associations have few new members discovered from recent observations at La Silla (ESO). Their final results will be presented very soon in a next paper. However, the introduction of these new members do not change significantly their mean curves (see below).

\section{Implications}

Fig. 1 shows the mean curves of the diagrams (Teff vs. Li abundance) of those associations. In da Silva et al. (2009) is shown that the errors of the Li abundance determination can not 


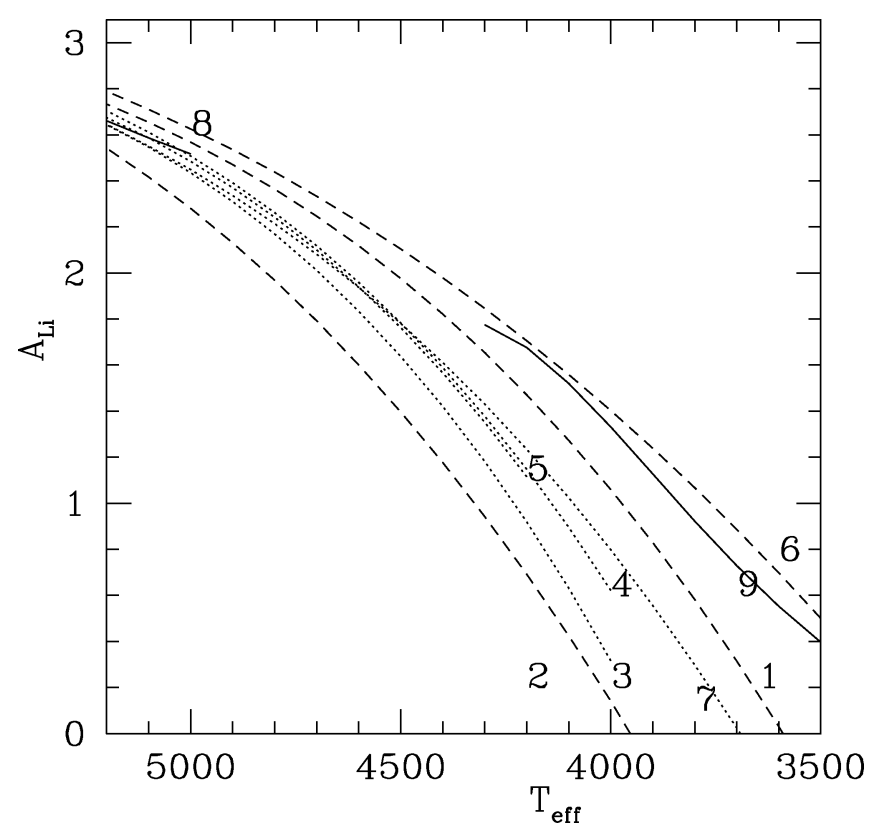

Figure 1. The mean curves for the young associations.

explain the dispersion of the points for a given Teff. Then, this dispersion is real. Stars with $\operatorname{vsin}(\mathrm{i})$ smaller than $20 \mathrm{~km} / \mathrm{s}$ are, in average, under those of vsin(i) larger, shown a larger $\mathrm{Li}$ depletion. The diagrams of the stellar $\mathrm{Li}$ abundances vs Teff corresponding to the program associations above show, for Teff $<5000 \mathrm{~K}$, a clear separation among the stars associations. As we can see on Fig. 1, the curve of $\epsilon \mathrm{ChA}$, what has $\sim 6 \mathrm{My}$, is upper the other curves; that of ColA, with $\sim 30 \mathrm{My}$, is in the middle and the curve corresponding to $\mathrm{AB}$ Dor, with $\sim 70 \mathrm{My}$, is below the other ones. In da Silva et al. (2009) we showed that the AB Dor stars are in the band corresponding to the Pleiades stars $(\sim 100 \mathrm{My})$, given one more evidence that those associations have the same age, what is in agreement with a recent 3D Galactic dynamical analysis indicating a common origin of these two groups (Ortega et al. 2007). We showed also the importance of the rotational velocity in the $\mathrm{Li}$ depletion: stars with larger rotational velocity have a Li depletion smaller than stars with smaller rotational velocity.

\section{References}

da Silva, L., Torres, C. A. O., Quast, G. R., de la Reza, R., Melo, C. H. F., \& Sterzik, M. 2009, $\mathrm{A} \& \mathrm{~A}$, in press

Kaufer, A., Stahl, O., Tubbesing, S., Norregaard, P., Avila, G., Francois, P., Pasquini, L., \& Pizzella, A., 1999, The Messenger 95, 8

Ortega, V. G., Jilinski, E., de la Reza, R., \& Bazzanella, B. Monthly Notices of the Royal Astronomical Society, 2007, Volume 377, 441

Torres,C. A. O., Quast, G. R., da Silva, L., de la Reza, R., Melo, C. H. F., \& Sterzik, M. 2006, A\&A, 460, 695

Torres, C. A. O., Quast, G. R., Melo, C. H. F., \& Sterzik, M. 2008, Handbook of Star forming Regions, Volume II: The Southern Sky ASP Monograph Publications, Vol. 5. Edited by Bo Reipurth, p 757 